\title{
A Review on Cloud Gaming
}

\author{
Sunanda D. Biradar ${ }^{1}$, Nusrathjabeen. Nagoor ${ }^{2}$ \\ Assistant Professor, Department of CSE, B.L.D.E.A ${ }^{e e}$ s Dr. P.G. Halakatti College of Engineering and Technology, \\ Vijayapur, Karnataka, India ${ }^{1}$
}

PG Scholar, Department of CSE, B.L.D.E.A ${ }^{e e}$ s Dr. P.G. Halakatti College of Engineeringand Technology, Vijayapur,

Karnataka, India ${ }^{2}$

\begin{abstract}
The present paper mainly focuses on literature review of cloud gaming. cloud gaming model it emerges with the advancement of communication technologies and cloud computing. Moving towards PCs, smartphones consoles tablets, people can have opportunity to use the games via data streaming, despite of the computing power of devices. The Internet is the style of communication between the game, and device hosted on a cloud environment. The big data analytics models it is an having ability to work with these data, and provides inputs for predictive actions and assertive analysis . A technique is shown in this study to indicate and analyzing the players' patterns in a virtual environment to optimize user experience. Using the visualization tools and Hadoop technology on a Cloud based cluster, it was possible to map and identify the players'behaviors as well as their gameplay patterns.
\end{abstract}

Keywords: Cloud Gaming; Big Data Analytics; Artificial Neural Networks.

\section{I.INTRODUCTION}

Cloud gaming is an online playing gaming system. video game is one of the most growing business in the entertainment industry, the mobile game includes candy crush and angry birds and many more, that are using nowdays.The increase in virtual entertainment on gadget such as smartphones, tablets is improving as the game developer companies are operating in the market in recent years. the utilization of games had a increase in growth as the promotion of new mobile technologies increased [1,2].

The different types of devices, like consoles computers, and smart TVs, prompts a evolution in the existing distribution offer of these games. In this scenario a concept emerges, trying to fulfill this demand for a accessible, online, hardware power independent and multi-device game, these elements are part of the Cloud Gaming model. The model implies that any device can access, via a client, the server on a Cloud remotely through the Internet, the servers process the information sent by the device and give the actions back via streaming [3], [4].

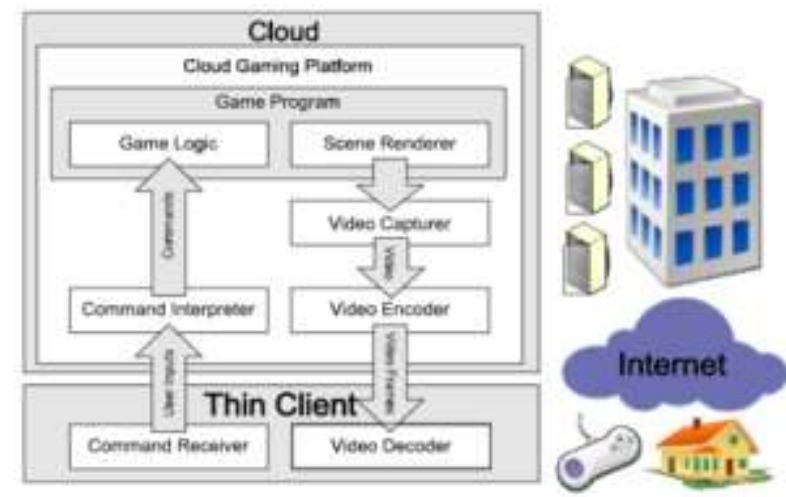

Fig:1 Typical cloud gaming services

The cloud gaming platform runs computer game programs, that can constitute as shown in fig 1: (i) Game logic it has a responsibility to convert the gamer commands into in-game interactions, and (ii) Game scenes are generated from scene renderer. The command interpreter where the game commands comes. and through the video capture where scenes are captured, and these scenes are compressed by encoder.

As we can see from the figure 1 the arrow marks at the bottom of the figure, through thin clients user inputs can be received for the gaming platform the gaming platform can send video frames to thin clients. in thin clients two 
components are necessary: a)video decoder. b)command receiver. the video decoder it can be thought of as inexpensive decoder chips that is produced. and command receiver, connection is made to game controller for example keyboard, mouse's joysticks etc.

There are tremendous advantages are there for cloud gaming to gamers, service providers ,game developers.

For gamers:- a) Access to the game anytime and anywhere. b) Can buy games on-demand c) There is no need to upgrade the hardware. d)It has features where we can see the ongoing tournaments and also share game reply's with people or friends.

For game developers:- a) Avoiding piracy because as the game software is never downloaded to client computer. b) For reducing the testing costs and porting concentrate on a single platform.

For service provider:- a) creates more demands on already deployed resources. b) Leads to new business model[5].

CloudUnion provided greater than two hundred games through its cloud platform in the year 2013. users can play games by using browser or downloading the CloudUnion's client. CloudUnion's policy bears game streaming in different qualities, ranging from $320 \times 240$ to $1024 \times 768$.

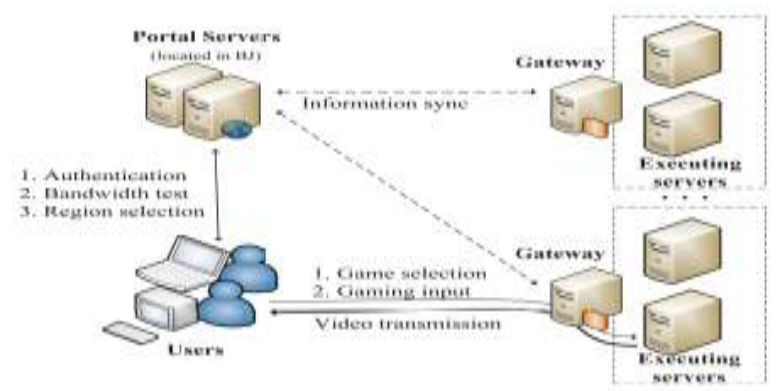

Fig 2. CloudUnion's architecture for cloud gaming platform

the bandwidth required is $2 \mathrm{Mb} / \mathrm{s}$, for clients. but with a $6 \mathrm{Mb} / \mathrm{s}$ or more high-speed connection is put forward for high quality game streaming. fig 2 shows the CloudUnion's infrastructure. A portal server accounts for authentication, user registration, and bandwidth test. when a user enters into the system, portal server will return a list of gateways that are positioned in different data centers, from which user can choose one to start gaming. when user selects a game that will be routed to gateway of near by region data center[6].

\section{WORLD OF WARCRAFT HISTORY}

The players behavior is one of the important factor for gaming system. it is a MMORP game that was released in 2004 as shown in fig 3 .WoW take place in 3 dimensional representation players can interact with through the characters.

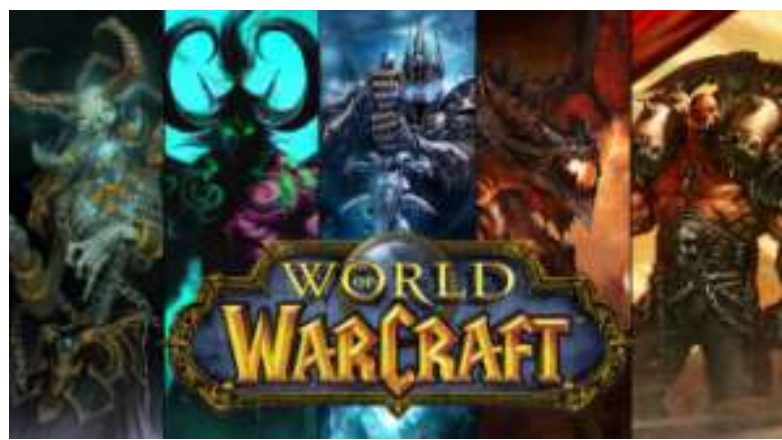

Fig 3: World of war craft

\section{A. Defining dataset}

The world of warcraft dataset consist of the records of 91065 avatars. and the data includes the attributes that is race class levels, gameplay times during 1107 days in the year from Jan 2006 to 2009.and it has 138084 log files that are divided into folders which represent 36 million records[6].

\section{B. Formulating dataset}

The log file has 12 different fields and a log containing a record was selected to train and test the neural network. the fields are null, query time, queryseqno, avatarID, guild, level class zones, race, record line no. 


\section{Artificial neural network}

The neural network is an interconnected group of nodes each circular node represent the neurons. a back propogation algorithm is used that is efficient for analyzing and identifying the pattern the network with 4 neurons is at input layer and seven neurons at the hidden layer and one at the output layer.

\section{RELATED WORK}

Anders et al[7]: Authors introduced the clustering of player behavior pattern that provides a way to locate behavioral pattern that are actionable for game developer. they also presented the case studies that mainly focuses on analysis of clustering that were applied to the high dimensional players behavior that covers a combined total of 260000 characters from commercial games such as MMORP game and battlefield. Simplex volume maximization it is applicable to large scale dataset k-means clustering were applied for two dataset that are combined taking into consideration of design for the games. that results into actionable behavioral pattern.

Depasquale et al.[8] presented a cloud gaming platform based on the RemoteFX extension of Windows remote desktop protocol. Modern Windows servers leverage GPUs and Hyper-V virtual machines to enable various remote applications, including cloud games. Their experiments reveal that RemoteFX allows Windows servers to better adapt to network dynamics, but still suffers from high frame loss rate and inferior responsiveness.

Yuri Demchenko and peter Membrey[9]: authors discussed the nature of big data that were originated from different industry, scientific and social activity domains. and also proposed the improved big data definition that include big data properties that are (volume, velocity, value, variety verciety).and data analytics, infrastructure and security. The big data architecture framework was proposed that addresses all aspect of the big data ecosystem. the main intension behind the proposed paper is to provide a consolidated view of the big data phenomena and related challenges to modern technologies.

T jeng et al[10]: presented the paper that recognizes the human behavior pattern in the game. the multilayer feedforward neural network with back propogation training algorithm with the architecture of four neurons in input layer and seven neurons in hidden layer and one neuron in output layer that is able to pattern in paper rock scissor game.

Wei cai et al: authors discussed cloud gaming model or system that host the game in the cloud environment, view and gamelpay are streamed to players terminal[5]. To address the high-bandwidth issue of real time gaming video transmission. For this issue they have represented the cloudlet-assisted multiplayer cloud gaming system. they have also used a mathematical formulation for the purpose of the studying player behaviors regarding cooperative sharing pattern for optimizing system performance in terms of user experience qualities. To reduce the computationally complexity an heuristic algorithm is proposed. the main intension behind this paper was to provide the high quality of experience compared with the existing platform with using adhoc cloudlet assistance.

Yussin et al[11]: In this proposed paper they have studied the problem of how to dispatch the play requests to the cloud server. they shown strategy for dispatching of players requests that affect the cost of total service for the cloud gaming system. this dispatching problem is the variant of the dynamic bin packing algorithm however these algorithm is not efficient in terms of resource usage. to address this issue they proposed the play request dispatching algorithm that assigns the request based an predicted end time of game sessions. the algorithm with neural network based prediction can minimizes the resources waste of servers and also decreases service cost when compared to FF and BF algorithms and also benefits are particularly significant for match based games.

Koun- Ta et al[12]: proposed the measurement technique for the evaluation of the Qos of cloud gaming system, they try to proved the effectiveness of their scheme using the case study that comprises two well known cloud gaming system that are streamMyGame and OnLive. Their results shown that the OnLive game has a better performance because that has a adaptable frame rates, and good graphics qualities and also less server processing delays that consumes less bandwidth. they also concentrated on some metrics that are traffic characteristics latency graphic quality. the main goal of their research was to provide best quality of service(QoS) for cloud gaming system.

\section{CONCLUSION}

The Cloud Gaming disputes are not only in the building of the model but also how to take out useful information of all structured and the unstructured data. that are generated for the improvements of the game and to understand players. purpose of this survey is to show how to take out or extract the information found on players characteristics action and 
also behaviors in online game environment through the neural networks and the Big data. by using big data it is able to map gameplay patterns by usage time. and also a heatmap is used to identify different zones based on avatars characteristics that support decision making.

\section{REFERENCES}

[1] Victor, Pollyana Program in Electrical and Computer Engineering Mackenzie Presbyterian University, UPM Sao Paulo, Brazil, 2016 IEEE International Conference on Big Data (Big Data).

[2] Fleury, Afonso, Davi Nakano, and J. H. D. O. Cordeiro. "Mapeamento da Indstria Brasileirae Global de Jogos Digitais."So Paulo: GEDIGames/USP, 2014.

[3] Xue, Zheng, et al. "Playing high-end video games in the cloud: a measurement study." IEEE Transactions on Circuits and Systems for Video Technology, v. 25, n. 12, p. 2013-2025,2015.

[4] Wallis, Thomas. "The challenges and opportunities for cloud in gaming." Cloud Gaming USA, 2015

[5] W. Cai "Survey on Cloud Gaming: Future of Computer Games" 2169-3536 2016 IEEE. Volume 42016.

[6] Yeng-Ting Lee, Kuan-Ta Chen, Yun-Maw Cheng, and Chin-Laung Lei, "World of Warcraft Avatar History Dataset" In Proceedings of ACM Multimedia Systems, 2011.

[7] Anders Drachen "Guns, Swords and Data: Clustering of Player Behavior in Computer Games in the Wild" 2012 IEEE Conference on Computational Intelligence and Games (CIG'12).

[8] ] E. Depasquale et al., "An analytical method of assessment of RemoteFX as a cloud gaming platform," in Proc. IEEE Conf. Medit. Electrotech.Conf. (MELECON), Beirut, Lebanon, Apr. 2014.

[9] Yuri Demchenko, Cees de Laat"

Defining Architecture Components of the Big Data Ecosystem" IEEE 2014.

[10] Tjeng Wawan Cenggoro.” Recognition of a Human Behavior Pattern in Paper Rock Scissor Game Using Backpropagation Artificial Neural Network Method"2014 $2^{\text {nd }}$ international conference on information and communication technology(ICoICT).

[11] Yusen Li, Xueyan Tang, Senior Member, IEEE." Play Request Dispatching for Efficient Virtual Machine Usage in Cloud Gaming" 2052 IEEE TRANSACTIONS ON CIRCUITS AND SYSTEMS FOR VIDEO TECHNOLOGY, VOL. 25, NO. 12, DECEMBER 2015.

[12] Kuan-Ta Chen, Member, IEEE." On the Quality of Service of Cloud Gaming Systems" IEEE TRANSACTIONS ON MULTIMEDIA, VOL. 16, NO. 2 FEBRUARY 2014. 